Thorax (1968), 23, 537.

\title{
Sternal sinus following Teflon reconstruction of the right ventricular outflow tract
}

\author{
P. B. DEVERALL AND G. H. WOOLER \\ From the Department of Thoracic Surgery, General Infirmary, Leeds
}

\begin{abstract}
A case of chronic sternal sinus is described in which exploration revealed a loose patch of Teflon felt which had been used three years earlier in reconstructing the outflow tract of the right ventricle in a case of congenital pulmonary stenosis. Teflon, a synthetic polymer of low biological reactivity, while being suitable in its solid sheet form for use in plastic reconstructive surgery, is not ideally suited to cardiovascular work. The properties of such an ideal material are defined. The low reactivity and porosity of Teflon, by reducing fibroblastic invasion, lessens the likelihood of the material being incorporated by host tissues. From experimental and clinical evidence we no longer regard Teflon felt as a suitable prosthetic material for use in intracardiac or vascular work or in reconstruction of the right ventricular outflow tract.
\end{abstract}

\section{CASE REPORT}

J. T. was first seen in 1958 at the age of 24 years, when he complained of shortness of breath and palpitations. A diagnosis of pulmonary stenosis was confirmed by catheterization and an angiocardiogram. Open pulmonary valvotomy was performed in November 1958 under moderate hypothernia with inflow occlusion. The pulmonary valve ring was small with cusp fusion but some relief of obstruction was obtained by rividing two commissures. After some improvement he developed further similar symptoms in 1960 and reinvestigation revealed a combination of valvular and infundibular obstruction. Re-exploration with cardiopulmonary bypass was carried out in February 1964. A midline sternal split access was difficult as the right ventricle was adherent to the back of the sternum. There was an infundibular stricture and a small pulmonary valve ring. A vertical ventriculotomy was extended across the valve ring, the infundibular stricture was resected, and the outflow tract reconstructed using a patch of Teflon felt with reinforcing Teflon pledgets at the suture line.

He did well after this operation, but presented after eight months with a discharging sinus at the lower end of the sternal incision. A penicillin-resistant staphylococcus was cultured from the discharge and a sinogram showed a narrow track extending deep to the sternum. Antibiotics were of no value. In December 1965 a second sinus developed on the left side of the sternum opposite the third costal cartilage. Wire sutures used in repairing the sternum were removed without benefit.

In April 1967 a third sinus appeared at the right border of the sternum at the same level as that on the left. A sinogram (Figs 1 and 2) showed a retro-
- sternal track, but in addition peripheral bronchial filling in the left upper lobe.

The sinuses were explored in July 1967 by excising the skin between them and then nibbling away the sternum to expose the track. A cavity deep to the sternum extended to the left and was being explored when strands of material were seen. On grasping this material the complete Teflon patch with pledgets and sutures (Fig. 3) was easily removed. The cavity was packed and on subsequent weekly occasions the wound was dressed and eventually it was resutured; complete healing occurred in about six weeks.

\section{DISCUSSION}

Wesolowski, Fries, and Sawyer (1961) summarized the specifications of cardiovascular prostheses as follows: :

1. Non-toxic, non-allergic

2. No deterioration on implantation: biological reactivity not critical

3. Porous

4. Low implant porosity, high healing poros.ty

5. Desirable mechanical characteristics

(a) able to be sutured

(b) crimped (for vessel prostheses)

(c) twistable.

Halpert, DeBakey, Jordan, and Henly (1960), following clinical experience with the use of prostheses and homografts in replacing human aorta, concluded that 'the ideal prosthetic material may consist of a synthetic material the fibres of 


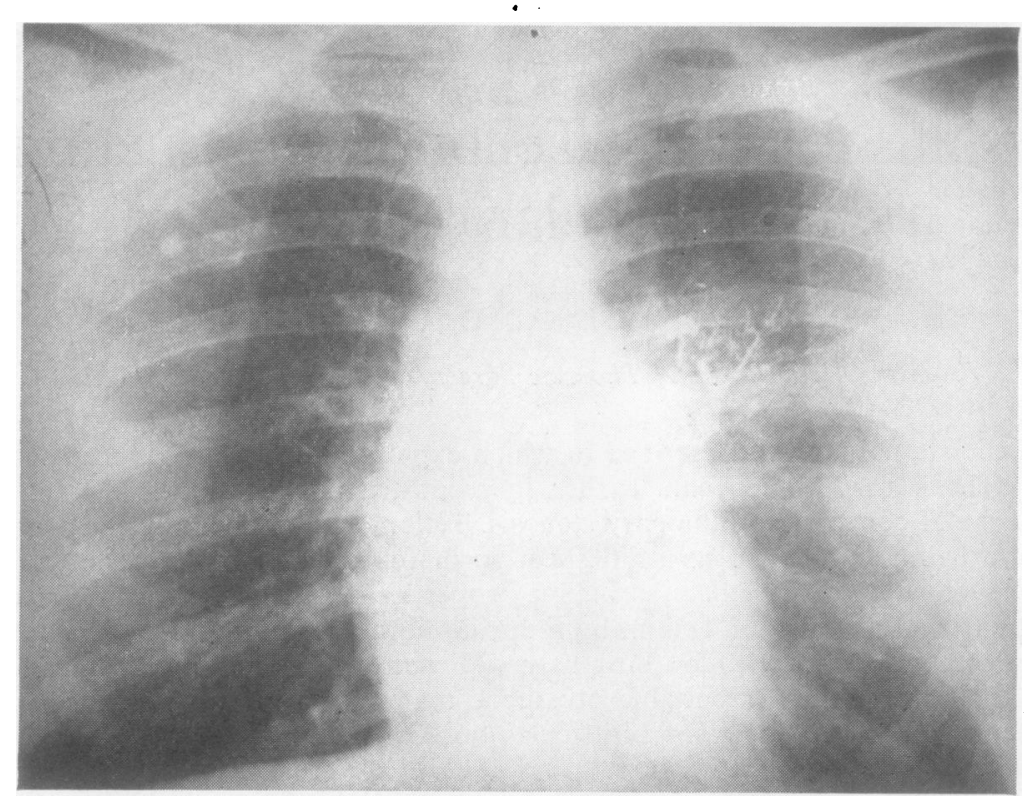

FIG. 1. Sinogram, 1967, showing bronchial filling of the left upper lobe, P.A. view.

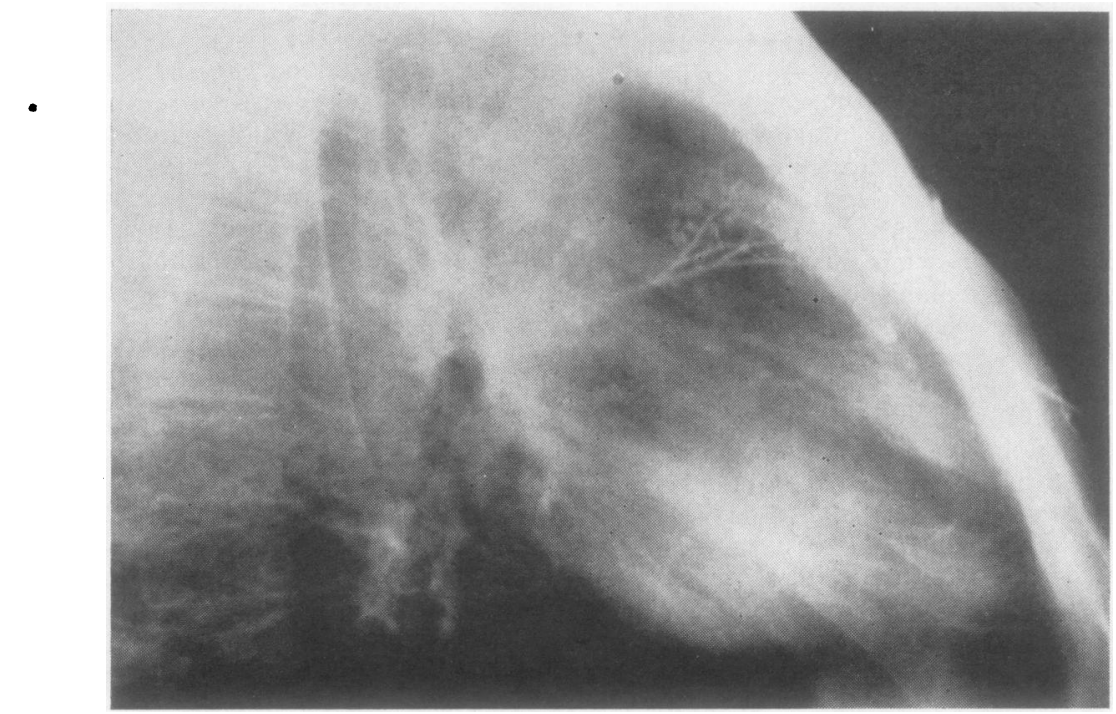

FIG. 2. Lateral film of sinogram, 1967. Retrosternal pocket with peripheral bronchial filling.

which are interspersed with absorbable haemostatic fibres woven or knitted tightly enough to prevent blood loss at the time of insertion. Later, when the haemostatic fibres are absorbed, sufficient porosity will occur to allow satisfactory

ingrowth of fibroblasts and endothelial cello resulting in attachment of the prosthesis to the host tissues.' covered by Plunkett (1941), is a synthetic polymer 


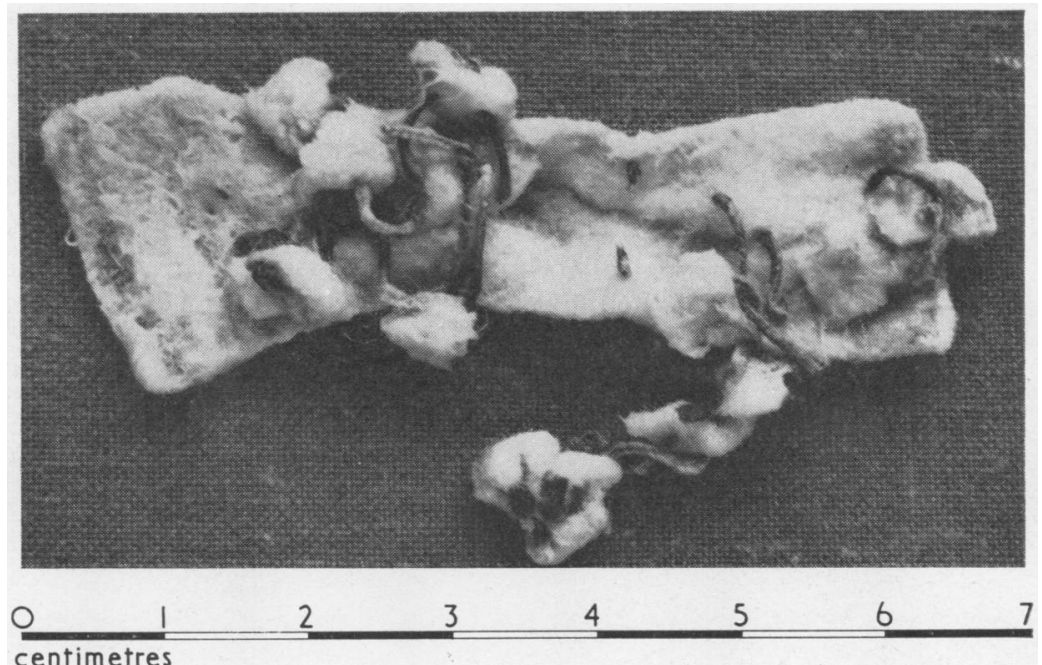

FIG. 3. Teflon patch with Teflon pledgets as removed in July 1967, three and a half years after insertion.

of low biological reactivity. In its sheet solid form, its use has become established in plastic surgery (Barron, Borchgrevink, and El Bayadi, 1966) and here its low reactivity has been of value. In vascular surgery incorporation of the prosthesis by fibroblastic and endothelial invasion is desirable, and it is the low reaction to Teflon and the failure of cellular invasion which have raised doubts as to its suitability in vascular work in contrast to a material such as Dacron. Roe (1961) warned that difficulties due to non-healing would be experienced with Teflon.

Sauvage, Gross, Rudolph, Pontius, and Watkins (1961) and Egerton and Windsor (1965) investigated the use of autogenous and prosthetic materials in reconstructing the outflow tract of the right ventricle. They described a generally poor though variable fibrous invasion of Teflon, though they agreed with Harrison's (1959) observation that tensile strength was retained, to be contrasted with the rapid breakdown of Ivalon and the loss by nylon of $80 \%$ of its strength in one year. Both groups of workers described the formation of a bland fibrous capsule, all of which observations are presumably related to low biological reactivity. Pericardium alone, or backed with Dacron, was recommended on grounds of its being haemostatic, suitable for manipulation and suture, and well incorporated by host tissues.

Wesolowski and others (1961) noted that calcification could occur at the edges of nonincorporated Teflon and found its occurrence to be inversely proportional to pore size. Variation in the latter probably explains the reported differences in fibrous invasion, the latter being facilitated by an increase in the pore dimension.

It seems likely that the 'non-wettability' characteristic of Teflon is a reflection of poor porosity. In lieu of the observations concerning calcification it is interesting to speculate on its occurrence following pulmonary artery banding using different types of material for the taping procedure.

It has been shown experimentally that a transfer of ions occurs across normal vessel walls and maintains an electro-negative charge on the luminal aspect. It is not clear what the repellent effect of this or similarly charged red blood cells may be, but the obstruction to ionic transfer with solid or non-porous prosthetic material may well be important.

Recently, Liotta, Hall, Akers, Villanueva, O'Neal, and DeBakey (1966) studied implantable blood pumps and noted that a deposition of blood protein occurred on the plastic surface at a blood plastic interface and this protein layer subsequently fragmented and embolized. Lining the plastic with nylon and Dacron velour prevented embolization, as the deposited fibrin became firmly adherent and subsequently covered by an endothelial-like cell layer. In the case of Teflon, a nearby unattached fibrin deposit was noted. These observations are also of interest in relation to Teflon and to prosthetic valves.

Payne and Kirklin (1961) reported aneurysmal dilatation of the right ventricular outflow tract using Ivalon. We have seen it following direct 
suture of a vertical ventriculotomy and with unsupported pericardium, but not with Teflon.

Though the presence of infection makes direct comparison impossible, this case is of interest in that the Teflon was unattached, its consistency and strength appeared unchanged, examination revealed no fibrous invasion, nor was calcification seen. There is no evidence of aneurysmal dilatation of this patient's right ventricular outflow tract.

\section{REFERENCES}

Barron, J. N., Borchgrevink, H., and El Bayadi, N. (1966). A report on Teflon. Brit. J. plast. Surg., 19, 113.

Egerton, W. S., and Windsor, M. F. (1965). Assessment of autogenous and prosthetic materials in the right ventricular outflow tract. Aust. N.Z. J. Surg., 35, 63.
Halpert, B., DeBakey, M. E., Jordan, G. L., and Henly, W. S. (1960) The fate of homografts and prostheses of the human aorta Surg. Gynec. Obstet., 111, 659. Harrison, J. H. (1959). Synthetic materials as vascular prostheses $\frac{\bar{\sigma}}{\overparen{D}}$
Ibid., 108, 433.

Liotta, D., Hall, C. W., Akers, W. W., Villanueva, A., O’Neal, R. M. and DeBakey, M. E. (1966). A pseudoendocardium for implant able blood pumps. Trans. Amer. Soc. artific. intern. Organs, 12 129.

Payne, W. S., and Kirklin, J. W. (1961). Late complications after plastic reconstruction of outflow tract in tetralogy of Fallot plastic reconstruction of outflow tract in tetralogy of Fallot $\vec{\omega}$. Surg., 154, 53.
Ann

Plunkett, R. J. (1941). U.S. patent no. 2,230,654.

Roe, B. B. (1961). Replacement of right ventricular myocardiume (Discussion). J. thorac. cardiovasc. Surg., 42, 780.

Sauvage, L. R., Gross, R. E., Rudolph, A. M., Pontius, R. G., and Watkins, E. (1961). Experimental study of tissue and prosthetic grafts with selected application to clinical intracardiac surgery: Ann. Surg., 153, 321.

Wesolowski, S. A., Fries, C. C., and Sawyer, P. N. (1961). The unimportance of biological reactivity of the synthetic vascularo graft wall. Trans. Amer. Soc. artific. intern. Organs, 7, 296. 